\title{
On the Decidability of Axiomatized Mereotopological Theories
}

\author{
Hsing-chien Tsai
}

\begin{abstract}
The signature of the formal language of mereotopology contains two predicates $P$ and $C$, which stand for "being a part of" and "contact," respectively. This paper will deal with the decidability issue of the mereotopological theories which can be formed by the axioms found in the literature. Three main results to be given are as follows: (1) all axiomatized mereotopological theories are separable; (2) all mereotopological theories up to ACEMT, SACEMT, or SACEMT $^{\prime}$ are finitely inseparable; (3) all axiomatized mereotopological theories except $\mathbf{S A X}, \mathbf{S A X} \mathbf{X}^{\prime}$, or $\mathbf{S} \overline{\mathbf{B}} \mathbf{X}^{\prime}$, where $\mathbf{X}$ is strictly stronger than CEMT, are undecidable. Then it can also be easily seen that all axiomatized mereotopological theories proved to be undecidable here are neither essentially undecidable nor strongly undecidable but are hereditarily undecidable. Result (3) will be shown by constructing strongly undecidable mereotopological structures based on two-dimensional Euclidean space, and it will be pointed out that the same construction cannot be carried through if the language is not rich enough.
\end{abstract}

\section{Introduction}

What is mereotopology? Such a term is ambiguous in the literature. Sometimes it means a logical theory, for example, general extensional mereotopology (GEMT; see Casati and Varzi [1, Chapter 4]). Sometimes it means a kind of algebraic structure, that is, a Boolean algebra under $\subseteq$ whose domain consists of some regular open sets of a given topological space (see Pratt-Hartmann [10, p. 18]). I will understand "mereotopology" in the first sense. But I will in a lot of places use "mereotopological theory" instead of "mereotopology" when referring to a theory of such kind. In this paper, I will deal with the decidability issue of mereotopological theories formed by axioms that can be found in the literature.

Received August 2, 2012; accepted January 22, 2013

2010 Mathematics Subject Classification: Primary 03C99; Secondary 06F99

Keywords: mereotopology, mereology, decidability, separability, finite separability

(C) 2015 by University of Notre Dame 10.1215/00294527-2864307 
Mereotopological theories are extensions of mereological theories, which are formed by axioms based on a binary predicate "being a part of." The mereological axioms which will be considered in this paper basically come from Simons [12] and [1], but many of them are in effect more up-to-date reformulations of axioms given by Leśniewski [6]. By adding a new binary predicate "contact" and some new axioms about such a predicate, mereological theories can be extended to mereotopological theories. This is what has been done in [1], and I will focus mainly on the axiomatized mereotopological theories given there. ${ }^{1}$

Now I will give a quick background of mereotopological theories. The signature of the first-order language of mereotopology consists of two binary predicates $P$ and $C$, which stand for "being a part of" and "contact," respectively. But for the sake of convenience of expressing complicated things, four additional predicates are defined as follows:

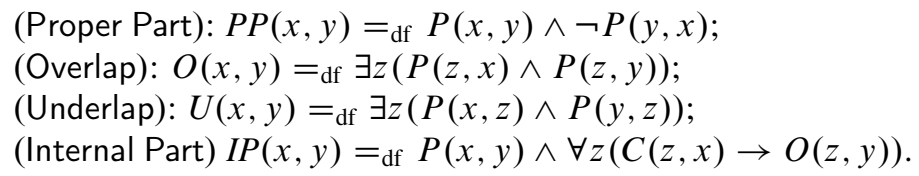

According to [1], there are two approaches to the formalization of mereotopological theories: one is to keep both $P$ and $C$ as primitives; the other, to define $P$ by using $C$. For the first approach, we have the following mereotopological axioms:

(C1) $C(x, x)$ (reflexivity for $C$ );

(C2) $C(x, y) \rightarrow C(y, x)$ (symmetry for $C$ );

(C3) $P(x, y) \rightarrow \forall z(C(z, x) \rightarrow C(z, y))$ (monotonicity);

(P1) $P(x, x)$ (reflexivity for $P$ );

(P2) $(P(x, y) \wedge P(y, x)) \rightarrow x=y$ (antisymmetry for $P)$;

(P3) $(P(x, y) \wedge P(y, z)) \rightarrow P(x, z)$ (transitivity for $P)$;

(EP) $\forall x \forall y(\exists z P P(z, x) \rightarrow(\forall z(P P(z, x) \leftrightarrow P P(z, y)) \rightarrow x=y))$ (extensionality);

(WSP) $\forall x \forall y(P P(x, y) \rightarrow \exists z(P P(z, y) \wedge \neg O(z, x)))$ (weak supplementation);

(SSP) $\forall x \forall y(\neg P(y, x) \rightarrow \exists z(P(z, y) \wedge \neg O(z, x)))$ (strong supplementation);

(FS) $\forall x \forall y(U(x, y) \rightarrow \exists z \forall w(O(w, z) \leftrightarrow(O(w, x) \vee O(w, y))))$ (finite sum);

(FP) $\forall x \forall y(O(x, y) \rightarrow \exists z \forall w(P(w, z) \leftrightarrow(P(w, x) \wedge P(w, y))))$ (finite product);

(G) $\exists x \forall y P(y, x)$ (the greatest member);

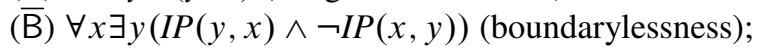

(C) $\forall x(\neg \forall z P(z, x) \rightarrow \exists z \forall w(P(w, z) \leftrightarrow \neg O(w, x)))$ (complementation);

(A) $\forall x \exists y(P(y, x) \wedge \forall z \neg P P(z, y))$ (atomicity);

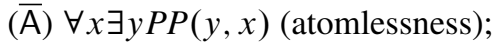

(UF) $\exists x \alpha(x) \rightarrow \exists z \forall y(O(y, z) \leftrightarrow \exists x(\alpha(x) \wedge O(y, x)))$ (unrestricted fusion axiom schema), for any formula $\alpha$ in which $x$ is free but $z$ and $y$ do not occur free. Note that $\alpha$ might have free variables other than $x .^{2}$

Intuitively, the $z$ in the formulation of UF is the object formed by "fusing" all the objects each of which has property $\alpha$. Now let us define the following function symbols: $:^{3}$ 
(Addition) $x+y=z$ iff $(U(x, y) \wedge \forall w(O(w, z) \leftrightarrow(O(w, x) \vee O(w, y)))) \vee$ $(\neg U(x, y) \wedge x=z)$;

(Product) $x \times y=z$ iff $(O(x, y) \wedge \forall w(P(w, z) \leftrightarrow(P(w, x) \wedge P(w, y)))) \vee$ $(\neg O(x, y) \wedge x=z)$;

(Complement) $\sim_{x}=z$ iff $(\neg \forall z P(z, x) \wedge \forall w(P(w, z) \leftrightarrow \neg O(w, x))) \vee$ $(\forall z P(z, x) \wedge x=z)$;

(Fusion) $F x(\alpha(x))=z$ iff $(\exists x \alpha(x) \wedge \forall y(O(y, z) \leftrightarrow \exists x(\alpha(x) \wedge O(y, x)))) \vee$ $(\neg \exists x \alpha(x) \wedge \forall y P(y, z))$, for any formula $\alpha$ in which $x$ is free but $z$ and $y$ do not occur free.

Note that the fusion function symbol $F x$ is actually a metafunction symbol which takes formulas as parameters. Hence formally, we will take $F x(\alpha(x))=z$ as the abbreviation of its definition. These function symbols are well defined if the theory considered has at least (P1), (P2), (P3), and (SSP) (the theory formed by these four axioms is called extensional mereology; see below), for then $\forall z(O(x, z) \rightarrow O(y, z)) \rightarrow P(x, y)$ will be a theorem and by (P2) and such a theorem, it is easy to see the uniqueness of the $z$ in each definition above. With the foregoing function symbols on hand, the following topological operators can be defined:

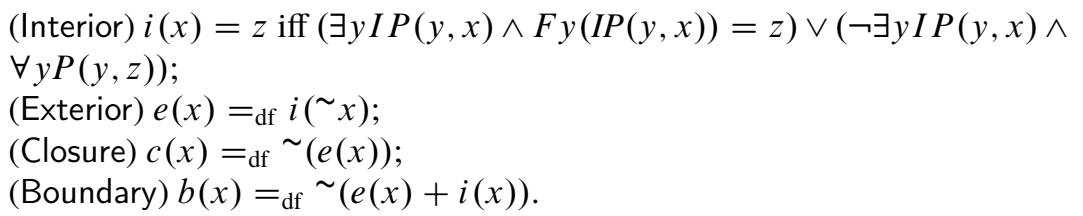

Then we may add three more axioms which are counterparts of Kuratowski's axioms for topological closures:

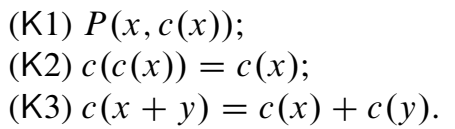

We will also adopt the following nomenclature of mereological and mereotopological theories given by [1]:

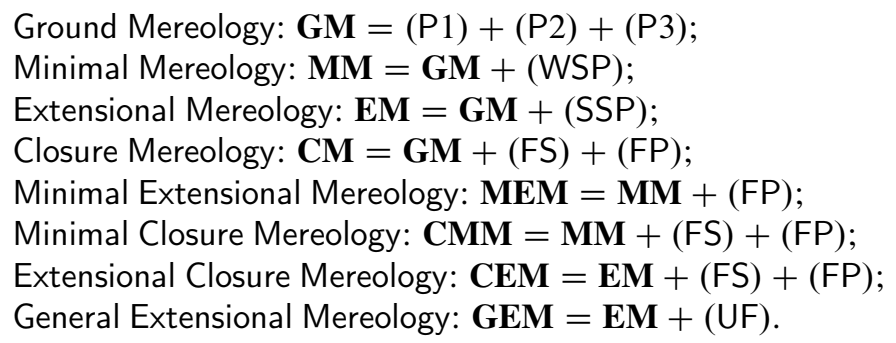

It is not difficult to see that GEM is the strongest theory on this list. As a matter of fact, it can be easily shown that GEM implies (EP), (WSP), (FS), $(F P),(G)$, and $(C)$. Now for any mereological theory $\mathbf{X}$ on the foregoing list, $\mathbf{X}+(\mathrm{C} 1)+(\mathrm{C} 2)+(\mathrm{C} 3)$ is named $\mathbf{X T}$ (if $\mathbf{X}$ is $\alpha$-mereology, $\mathbf{X T}$ will be read as $\alpha$-mereotopology; for instance, EMT is extensional mereotopology). For any $\mathbf{X T}$, $\mathbf{X T}+(\mathrm{A})$ is named AXT, XT $+(\bar{B})$ is named $\overline{\mathbf{B}} \mathbf{X T}$, and $\mathbf{X T}+(\overline{\mathrm{A}})$ is named $\overline{\mathbf{A} X T}$. Besides, GEMT $+(\mathrm{K} 1)+(\mathrm{K} 2)+(\mathrm{K} 3)$ is named GEMTC, GEMTC $+(\mathrm{A})$

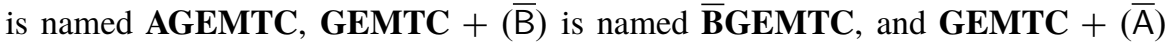


is named $\overline{\mathbf{A}}$ GEMTC. ${ }^{4}$ In this setting, there are two incompatible maximally consistent mereotopological theories AGEMTC and BGEMTC (it is easy to see that $\overline{\mathbf{B}}$ GEMTC implies $\overline{\mathbf{A}}$ and that no finite model can satisfy $\overline{\mathbf{B}}$ GEMTC). Later in Theorem 2.1 we will give models which witness their consistency.

For the second approach, besides the mereotopological axioms listed above, there is one more as follows:

(C4) $\forall z(C(x, z) \rightarrow C(y, z)) \rightarrow P(x, y)$ (the Converse of C3).

Then for any theory $\mathbf{X}$ in the first approach, $\mathbf{X}+(\mathrm{C} 4)$ is named $\mathbf{S X}$ (Strong $\mathbf{X}$ ). Note that (C3) and (C4) together means that $P$ can be defined by using $C$. Now there is a variation due to Clarke [3], which defines fusion by "contact" instead of "overlap." Such a variation replaces principles (FS), (FP), (C), and (UF) by the following versions (then all the function symbols defined above will also have to be redefined accordingly):

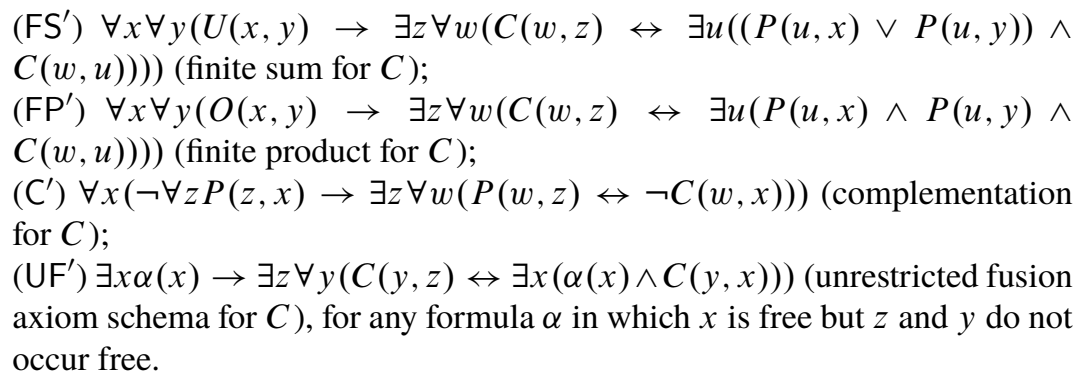

For any theory $\mathbf{S X}$ in the second approach, if we replace the occurrences (if any) of (FS), (FP), (C), and (UF), respectively, by $\left(\mathrm{FS}^{\prime}\right),\left(\mathrm{FP}^{\prime}\right),\left(\mathrm{C}^{\prime}\right)$, and $\left(\mathrm{UF}^{\prime}\right)$, the resultant

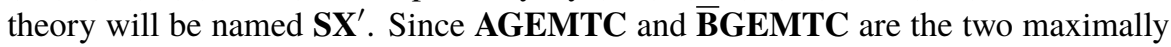
consistent theories in the first approach, it should be clear that the two maximally consistent theories which can be formed in the second approach are SAGEMTC and $\mathbf{S B G E M T C}$ (or SAGEMTC' ${ }^{\prime}$ and $\mathbf{S B}$ GEMTC ${ }^{\prime}$ if we adopt the said variation). Obviously no finite model can satisfy $\mathbf{S} \overline{\mathbf{B}} \mathbf{G E M T C}$ or $\mathbf{S} \overline{\mathbf{B}} \mathbf{G E M T C} \mathbf{C}^{\prime}$. However, in Theorem 2.1 we will construct a model which satisfies both theories.

The foregoing sets up the scope of mereotopological theories that I will deal with. Though naturally I will be mainly concerned with those theories with names, due to the nature of the method which I will adopt, as we will see, the results to be given will also cover the cases of other possible consistent combinations of the axioms in either approach.

As mentioned earlier, this paper will deal with the decidability issue of axiomatized mereotopological theories. More precisely, I will actually consider three properties: separability, finite separability, and decidability. The first two properties are defined as follows (the versions presented here are paraphrases of definitions given in Monk [8]).

(Effectively inseparable) Two sets $A$ and $B$ of natural numbers are effectively inseparable if and only if they are disjoint and there is a binary recursive function $f$ such that for any two disjoint recursively enumerable sets $C \supseteq A$ and $D \supseteq B$, $f(c, d) \notin C \cup D$, where $c$ and $d$ are indices of $C$ and $D$, respectively.

(Inseparable) A theory $T$ in a language $L$ is inseparable if and only if the set of Gödel numbers of the theorems of $T$ and the set of Gödel numbers of the sentences whose negations are theorems of $T$ are effectively inseparable. 
(Finitely inseparable) A theory $T$ in a language $L$ is finitely inseparable if and only if the set of Gödel numbers of the valid sentences in $L$ and the set of Gödel numbers of the sentences each of which can be refuted by some finite model of $T$ are effectively inseparable.

Of course, a theory is separable if it is not inseparable, and a theory is finitely separable if it is not finitely inseparable. The following is a list of facts concerning these properties (here we will assume that all theories considered are consistent).

(i) Decidability implies both separability and finite separability.

(ii) If a theory $T$ is finitely inseparable, then any theory in the same language which is weaker than $T$ will also be finitely inseparable; that is, for theories in the same language, finite inseparability is closed downwards.

(iii) If theories $T_{1}$ and $T_{2}$ are in the same language and $T_{2}$ is a finite extension of $T_{1}$, then the decidability of $T_{1}$ will imply the decidability of $T_{2}$.

(iv) If all theories considered are in the same language or the language of a weaker theory is included in the language of a stronger one, then inseparability is closed upwards.

(v) If a theory has finite models, it cannot be inseparable, for the theory of a finite model must be decidable. On the other hand, if a theory does not have finite models, it cannot be finitely inseparable. Hence any theory must be separable or finitely separable.

Although this paper is mainly concerned with the aforementioned three properties, as we will see, based on the proofs to be given, some questions concerning other properties relevant to decidability can also be answered. Keep in mind that, unless deliberately specified, all mereotopological theories considered in the following are those consistent ones which can be formed by using axioms in the first approach or in the second approach listed above.

\section{Separability and Finite Separability}

I will first give the following general result of separability.

\section{Theorem 2.1 All mereotopological theories are separable.}

Proof If a mereotopological theory has finite models, then it is obviously separable, for the theory of a finite mereotopological structure must be decidable. Observe that any mereotopological structure $\mathcal{M}$ whose domain has only one member which contacts itself and which is a part of itself will satisfy AGEMTC, SAGEMTC, and SAGEMTC' (of course, this also shows that all these theories are consistent). In this light, if all models of a mereotopological theory are infinite, then it must have $(\bar{A})$ or $(\bar{B})$. However, as mentioned earlier, for the first approach, $\overline{\mathbf{B}}$ GEMTC is the strongest among the theories each of which has no finite model, and for the second approach, the strongest is SB̈GEMTC or

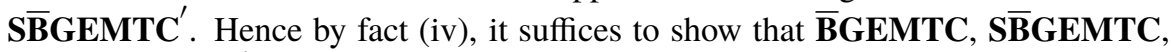
and $\mathbf{S} \overline{\mathbf{B}} \mathbf{G E M T C} \mathbf{C}^{\prime}$ are separable. Now construct a mereotopological structure $\mathcal{M}$ as follows: $\operatorname{Dom}(\mathcal{M})$ (the domain of $\mathcal{M})=\{x \subseteq \omega: x$ is nonempty periodic, that is, $x \neq \emptyset$ and $\exists n \in \omega \forall y(y \in x \leftrightarrow y+n \in x)\}, C^{\mathcal{M}}=\{(x, y) \in \operatorname{Dom}(\mathcal{M}) \times$ $\operatorname{Dom}(\mathcal{M}): x \cap y \neq \emptyset\}$, and $P^{\mathcal{M}}=\{(x, y) \in \operatorname{Dom}(\mathcal{M}) \times \operatorname{Dom}(\mathcal{M}): x \subseteq y\}$. Note that even in the second approach, $\operatorname{Dom}(\mathcal{M})$ will guarantee that the interpretation of $P$ must be set inclusion if $C$ is interpreted as nonempty intersection (it is easy to 
show that in the said model, if every set which intersects $x$ also intersects $y$, then $x$ will be a subset of $y$; see facts (a), (b), and (c) below). It is not difficult to see that $\mathcal{M}$ satisfies (C1), (C2), (C3), (C4), (P1), (P2), (P3), and (SSP). All these cases either follow immediately from relevant definitions or can be easily shown by using the following three facts: (a) any nonempty periodic proper subset of $\omega$ is infinite and not cofinite (note that $\omega$ itself is trivially nonempty periodic), (b) if $x$ is a nonempty periodic proper subset of $\omega$, then $\omega-x$ is also nonempty periodic, and (c) if $x$ and $y$ are nonempty periodic subsets of $\omega$, then $x \cap y$ is either the empty set or a nonempty periodic subset of $\omega$. Also observe that $C(x, y)$ and $O(x, y)$ are equivalent in $\mathcal{M}$ and hence (UF) is equivalent to (UF') in $\mathcal{M}$. Based on the foregoing observations, in order to show that $\mathcal{M}$ satisfies $\overline{\mathbf{B}}$ GEMTC, $\mathbf{S} \overline{\mathbf{B}}$ GEMTC, and $\mathbf{S} \overline{\mathbf{B}}$ GEMTC ${ }^{\prime}$, it suffices to show that (UF), ( $\overline{\mathrm{B}}),(\mathrm{K} 1),(\mathrm{K} 2)$, and (K3) are satisfied by $\mathcal{M}$. Since $C$ is equivalent to $O$ in $\mathcal{M}$, which means that $C$ can be defined in $\mathcal{M}$ by using $P, \mathcal{M}$ can be viewed as a mereological structure. Now consider a mereological theory $\mathbf{C E M}+(\mathrm{G})+(\mathrm{C})+$ $(\overline{\mathrm{A}})$. We have already known that $\mathcal{M}$ satisfies (P1), (P2), (P3), and (SSP). Obviously, $\mathcal{M}$ also satisfies (G) and by the aforementioned facts (a), (b), and (c), $\mathcal{M}$ satisfies (FS), (FP), and (C) as well. Furthermore, $\mathcal{M}$ satisfies $(\overline{\mathrm{A}})$, for it is trivial that any nonempty periodic subset of $\omega$ will have a proper subset which is also nonempty periodic. Due to these facts, $\mathcal{M}$ is a model of CEM $+(G)+(C)+(\bar{A})$. However, it is known that such a theory is complete (see Tsai [16]), so it will be equivalent to any of its consistent extensions, in particular, CEM $+(\mathrm{UF})+(\overline{\mathrm{B}})+(\mathrm{K} 1)+(\mathrm{K} 2)+(\mathrm{K} 3)$, where each occurrence of $C(x, y)$ will be replaced by $O(x, y)$ (note that if $C(x, y)$ is replaced by $O(x, y), I P x y$ is equivalent to $P x y$; hence $(\overline{\mathrm{B}})$ is equivalent to $(\overline{\mathrm{A}})$ and $i(x)$ is just $x$ itself). Therefore, $\mathcal{M}$ also satisfies (UF), ( $\overline{\mathrm{B}}),(\mathrm{K} 1),(\mathrm{K} 2)$, and (K3),

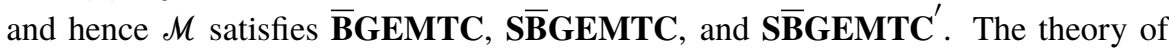
$\mathcal{M}$ is exactly CEM $+(\mathrm{G})+(\mathrm{C})+(\overline{\mathrm{A}})+\forall x \forall y(C(x, y) \leftrightarrow O(x, y))$, which is decidable, for it is a complete recursively axiomatized theory. But since $\mathcal{M}$ satisfies $\bar{B}$ GEMTC, SBGEMTC, and SBGEMTC ${ }^{\prime}$, the theory of $\mathcal{M}$ is a super-theory of

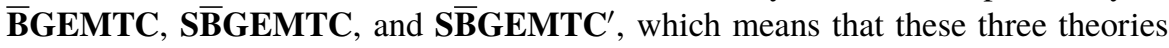
are separable.

The trick of the foregoing proof is to "collapse" a mereotopological structure into a mereological one by defining "contact" as "overlap." The same trick will be utilized again below, for then we can resort to some known results about the decidability of mereological theories. Before going on further, we will first introduce the following lemma which is very useful for proving the finite inseparability of a theory.

Lemma 2.2 Let $T$ and $T^{\prime}$ be two theories in languages $L$ and $L^{\prime}$, respectively. Assume that $L$ has only finitely many function symbols. Suppose that $L$ can be interpreted into a finitely axiomatized $L^{\prime}$-theory $S^{\prime}$. Call this interpretation I. If for each finite model $\mathcal{A}$ of $T$, there is a finite model $\mathscr{B}$ of $T^{\prime} \cup S^{\prime}$ such that $\mathcal{A}=\mathscr{B}^{I}\left(\mathcal{B}^{I}\right.$ is the L-structure defined in the $L^{\prime}$-structure $\mathscr{B}$ by using interpretation I) and $T$ is finitely inseparable, then $T^{\prime}$ is finitely inseparable too. ${ }^{5}$

Theorem 2.3 All mereotopological theories up to ACEMT or SACEMT or SACEMT' are finitely inseparable. 
Proof It is known that CEM is finitely inseparable (see Tsai [15]). Now, in view of Lemma 2.2, ACEMT, SACEMT, and SACEMT' will be finitely inseparable if every finite model of CEM can be in a definable way extended to a finite model which satisfies some finite extensions of ACEMT, SACEMT, and SACEMT' ${ }^{\prime}$ (note that any finite mereotopological structure must be atomic). Consider $S_{1}=$ ACEMT $+\forall x \forall y(C(x, y) \leftrightarrow O(x, y)), S_{2}=$ SACEMT + $\forall x \forall y(C(x, y) \leftrightarrow O(x, y))$, and $S_{3}=$ SACEMT $^{\prime}+\forall x \forall y(C(x, y) \leftrightarrow O(x, y))$. It is easy to see that under EM $+\forall x \forall y(C(x, y) \leftrightarrow O(x, y))$, (FS) and (FP) are equivalent, respectively, to $\left(\mathrm{FS}^{\prime}\right)$ and $\left(\mathrm{FP}^{\prime}\right)$. Hence $S_{2}$ and $S_{3}$ are equivalent and we only have to consider one of them, say, $S_{2}$. Now for any finite model $\mathcal{M}$ of CEM, it is easy to see that there are models $\mathcal{M}_{1}$ and $\mathcal{M}_{2}$ of $S_{1}$ and $S_{2}$, respectively, such that $\mathcal{M}=\mathcal{M}_{1}^{I}$ and $\mathcal{M}=\mathcal{M}_{2}^{I}$, where $I$ is the identity interpretation, that is, $I$ interprets " $\forall$ " (the domain) as " $x=x$ " and " $P$ " as " $P(x, y)$." This is because if we expand $\mathcal{M}$ by letting $C^{\mathcal{M}}=\{(x, y) \in \operatorname{Dom}(\mathcal{M}) \times \operatorname{Dom}(\mathcal{M})$ : for some $z \in \operatorname{Dom}(\mathcal{M}),(z, x) \in$ $P^{\mathcal{M}}$ and $\left.(z, y) \in P^{\mathcal{M}}\right\}$, the expanded model obviously satisfies (C1), (C2), (C3), and (C4), and therefore satisfies both $S_{1}$ and $S_{2}$. Then by Lemma 2.2, ACEMT, SACEMT, and SACEMT ${ }^{\prime}$ are finitely inseparable, and by fact (ii), all their subtheories are finitely inseparable too.

It is known that CEM $+(C)$ is decidable and CEM $+(G)$ is finitely separable (see Tsai [14]) and that GEM is decidable (see [16]). Hence, for theories strictly stronger than ACEMT or SACEMT, it is unlikely that we can utilize the same trick as above. However, I am going to show in the following that all mereotopological theories except those of the form $\mathbf{S A X}$ or $\mathbf{S A X} \mathbf{X}^{\prime}$ or $\mathbf{S} \overline{\mathbf{B}} \mathbf{X}^{\prime}$, where $\mathbf{X}$ is strictly stronger than CEMT, are undecidable even if their mereological counterparts are decidable (it is unknown whether a theory of any of the said forms is decidable or not). This means that the question whether a mereotopological theory strictly stronger than ACEMT or SACEMT or SACEMT' ${ }^{\prime}$ is finitely inseparable or not will still remain open.

\section{Strong Undecidable Mereotopological Structures}

A structure $\mathcal{M}$ of a language $L$ is strongly undecidable if and only if any $L$-theory which is satisfied by $\mathcal{M}$ is undecidable. Now in order to show the undecidability of mereotopological theories, I will construct two strongly undecidable mereotopological structures: one for the atomic mereotopological theories up to AGEMTC and the other for the atomless mereotopological theories up to $\bar{B}$ GEMTC, SBGEMTC, or $\mathbf{S B} \mathbf{C E M T}^{\prime}$. More precisely, consider the two-dimensional Euclidean space $R^{2}$ with the standard topology on it. Let us construct two mereotopological structures $\mathcal{M}_{1}$ and $\mathcal{M}_{2}$ as follows:

$$
\begin{aligned}
\operatorname{Dom}\left(\mathcal{M}_{1}\right) & \left.=\mathcal{P}\left(R^{2}\right) \text { the power set of } R^{2}\right) \backslash\{\emptyset\},{ }^{6} \\
P^{\mathcal{M}_{1}} & =\left\{(x, y) \in \operatorname{Dom}\left(\mathcal{M}_{1}\right) \times \operatorname{Dom}\left(\mathcal{M}_{1}\right): x \subseteq y\right\}, \quad \text { and } \\
C^{\mathcal{M}_{1}} & =\left\{(x, y) \in \operatorname{Dom}\left(\mathcal{M}_{1}\right) \times \operatorname{Dom}\left(\mathcal{M}_{1}\right): \operatorname{cl}(x) \cap \operatorname{cl}(y) \neq \emptyset\right\},
\end{aligned}
$$

where $\mathrm{cl}$ is the closure operator induced by the standard topology on $R^{2}$;

$$
\begin{aligned}
\operatorname{Dom}\left(\mathcal{M}_{2}\right) & =\mathcal{R} \mathcal{O}\left(R^{2}\right)\left(\text { the set of the regular open subsets of } R^{2}\right) \backslash\{\emptyset\} \quad \text { and } \\
C^{\mathcal{M}_{2}} & =\left\{(x, y) \in \operatorname{Dom}\left(\mathcal{M}_{2}\right) \times \operatorname{Dom}\left(\mathcal{M}_{2}\right): \operatorname{cl}(x) \cap \operatorname{cl}(y) \neq \emptyset\right\}
\end{aligned}
$$


where $\mathrm{cl}$ is the closure operator induced by the standard topology on $R^{2}$. Note that the interpretation of $P$ in $\mathcal{M}_{2}$ will also be the set inclusion, that is,

$$
P^{\mathcal{M}_{2}}=\left\{(x, y) \in \operatorname{Dom}\left(\mathcal{M}_{2}\right) \times \operatorname{Dom}\left(\mathcal{M}_{2}\right): x \subseteq y\right\},
$$

for it can be easily shown in $\mathcal{R O}\left(R^{2}\right)$ that if for any $z, \operatorname{cl}(z) \cap \operatorname{cl}(x) \neq \emptyset$ implies that $\operatorname{cl}(z) \cap \operatorname{cl}(y) \neq \emptyset$, then $x \subseteq y{ }^{7}$

I will first show that AGEMTC is satisfied by $\mathcal{M}_{1}$ and that $\bar{B}$ GEMTC,

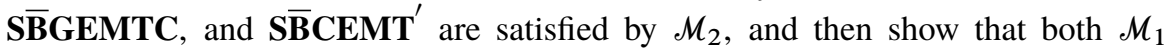
and $\mathcal{M}_{2}$ are strongly undecidable.

Lemma $3.1 \quad$ (a) $\mathcal{M}_{1}$ as defined above satisfies AGEMTC, and (b) $\mathcal{M}_{2}$ as defined

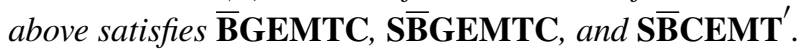

Proof For (a), by the interpretations of $P$ and $C$, it is trivial that $\mathcal{M}_{1}$ satisfies (P1), (P2), (P3), (C1), (C2), (C3), and (SSP). Since any set of a single point is in $\operatorname{Dom}\left(\mathcal{M}_{1}\right), \mathcal{M}_{1}$ is of course atomic and hence satisfies $(\mathrm{A})$. The structure $\mathcal{M}_{1}$ satisfies (UF) since any subset of $R^{2}$ except the empty set is in $\operatorname{Dom}\left(\mathcal{M}_{1}\right)$. As to (K1), $(\mathrm{K} 2)$, and (K3), first observe that for any $x \in \operatorname{Dom}\left(\mathcal{M}_{1}\right)$, if $x \neq R^{2}, \sim_{x}$ is indeed the complement under $R^{2}$, that is, $\sim_{x}=R^{2} \backslash x$, and that for any $x, y \in \operatorname{Dom}\left(\mathcal{M}_{1}\right)$, $x+y=x \cup y .{ }^{8}$ Then we will show that $c(x)$ is indeed the closure of $x$ under the standard topology of $R^{2}$, and it will be clear that $\mathcal{M}_{1}$ satisfies $(\mathrm{K} 1),(\mathrm{K} 2)$, and (K3). Now suppose that $x \in \operatorname{Dom}\left(\mathcal{M}_{1}\right), x \neq R^{2}$, and $x$ has nonempty interior; then we will show that $i(x)$ is indeed the interior of $x$. Let $u$ be the interior of $x$, that is, $u$ is the union of all open sets each of which is included in $x$. Obviously, $u \in \operatorname{Dom}\left(\mathcal{M}_{1}\right)$. In order to show that $i(x)=u$, it suffices to show that for all $s \in \operatorname{Dom}\left(\mathcal{M}_{1}\right), s$ overlaps $u$ if and only if there is some $z$ such that $\operatorname{IP}(z, x)$ and $z$ overlaps $s$ (see endnote 8). Consider any $s \in \operatorname{Dom}\left(\mathcal{M}_{1}\right)$. If $s$ overlaps $u$, then choose a point $p \in s \cap u$ and obviously $\{p\}$ is an internal part of $x$. Conversely, suppose that there is some $z$ such that $I P(z, x)$ and $z$ overlaps $s$. Now observe two facts. First, $z$ cannot consist of only some boundary points of $x$, for otherwise, the complement of $x$ will contact $z$ but will not overlap $x$, which contradicts the assumption that $z$ is an internal part of $x$. Second, if $\operatorname{IP}(z, x)$ and $P(y, z)$, then $\operatorname{IP}(y, x)$, for otherwise, there is some $u$ such that $C(u, y)$ and $\neg O(u, x)$, but $C(u, z)$ since $P(y, z)$, which contradicts $I P(z, x)$. Based on the foregoing two facts, $z \cap s$ is an internal part of $x$ and hence must contain some nonboundary points of $x$, which implies that $s$ must overlap the interior of $x$, that is, $u$. Now consider any $x \in \operatorname{Dom}\left(\mathcal{M}_{1}\right)$. There are three possibilities: first, $x=R^{2}$; second, $x \subset R^{2}$ and $\sim_{x}$ has nonempty interior; third, $x \subset R^{2}$ and $\sim x$ has empty interior (i.e., $x$ is dense). If $x=R^{2}$, by definition, $\sim_{x}=R^{2}, i(\sim x)=e(x)$ is also $R^{2}$, and $\sim_{e}(x)=c(x)$ is again $R^{2}$. If $x \subset R^{2}$ and $\sim x$ has nonempty interior, by what has been shown above, $e(x)=i(\sim x)$ is indeed the interior of the complement of $x$ and hence $c(x)=\sim e(x)$ is indeed the closure of $x$. If $x \subset R^{2}$ and $\sim x$ has empty interior, $e(x)=i\left(\sim_{x}\right)=R^{2}$ and hence $c(x)=\sim_{e}(x)=R^{2}$. Therefore, in any case, $c(x)$ is indeed the closure of $x$. However, it is known that $R^{2}$ with the standard topology on it satisfies Kuratowski closure axioms; hence $\mathcal{M}_{1}$ satisfies (K1), (K2), and (K3).

For (b), it is trivial that $\mathcal{M}_{2}$ satisfies (P1), (P2), (P3), (C1), (C2), (C3), (C4), and (SSP). To see that $\mathcal{M}_{2}$ satisfies $(\bar{B})$, observe that any nonempty regular open subset $x$ of $R^{2}$ has a nonempty proper subset, say, $y$, which is also regular open, and whose closure is included in $x$ (recall that $R^{2}$ is compact Hausdorff), and then it is easy to 
show that $I P(y, x)$ but $\neg I P(x, y)$. As for (UF), consider any nonempty subset $S$ of $\operatorname{Dom}\left(\mathcal{M}_{2}\right)$. Take the interior of the closure of $\bigcup S$ and call it $s$. Then it is easy to see that, for any $z \in \operatorname{Dom}\left(\mathcal{M}_{2}\right), z$ overlaps $s$ if and only if $z$ overlaps some member of $S$. Hence $\mathcal{M}_{2}$ satisfies (UF). Now we will show that $\mathcal{M}_{2}$ satisfies (K1), (K2), and $(\mathrm{K} 3)$, and it suffices to show that for any $x \in \operatorname{Dom}\left(\mathcal{M}_{2}\right), c(x)=x$. Observe that for any $x, y \in \operatorname{Dom}\left(\mathcal{M}_{2}\right)$, if $x$ overlaps $y$, then their intersection is also regular open. From this fact and the fact that $R^{2}$ is compact Hausdorff, it is easy to see that for any $x \in \operatorname{Dom}\left(\mathcal{M}_{2}\right), i(x)=x$. So if $x=R^{2}$, by the definition of " $c$ ", trivially $c(x)=x$. Otherwise, $x$ is a regular open proper subset of $R^{2}$. Then $R^{2} \backslash x$ must have regular open interior. Hence $e(x)=i(\sim x)$ is the interior of $R^{2} \backslash x$ and then $c(x)=\sim e(x)$ is $x$ again. Therefore, for any $x \in \operatorname{Dom}\left(\mathcal{M}_{2}\right), c(x)=x$. The

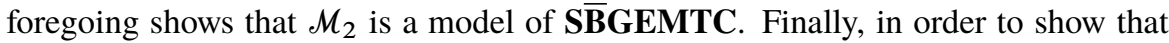
$\mathcal{M}_{2}$ is a model of $\mathbf{S B} \mathbf{C E M T} \mathbf{T}^{\prime}$, it suffices to show that $\mathcal{M}_{2}$ satisfies $\left(\mathrm{FS}^{\prime}\right)$ and $\left(\mathrm{FP}^{\prime}\right)$. We will argue that for any $x, y \in \operatorname{Dom}\left(\mathcal{M}_{2}\right)$, (i) the interior of the closure of $x \cup y$ will be the $z$ such that $\forall w(C(w, z) \leftrightarrow \exists u((P u x \vee P u y) \wedge C w u))$, and (ii) if $x$ overlaps $y, x \cap y$ will be the z such that $\forall w(C w z \leftrightarrow \exists u(P u x \wedge P u y \wedge C w u))$. Obviously, the closure of the interior of the closure of $x \cup y$ is just the closure of $x \cup y$. Since $\operatorname{cl}(x \cup y)=\operatorname{cl}(x) \cup \operatorname{cl}(y)$, for any $w, \operatorname{cl}(w) \cap \operatorname{cl}(x \cup y) \neq \varnothing$ if and only if either $\operatorname{cl}(w) \cap \operatorname{cl}(x) \neq \emptyset$ or $\operatorname{cl}(w) \cap \operatorname{cl}(y) \neq \emptyset$, and then (i) follows immediately. Now suppose that $x$ overlaps $y$. Since $\operatorname{cl}(x \cap y)=\operatorname{cl}(x) \cap \operatorname{cl}(y)$, for any $w, \operatorname{cl}(w) \cap \operatorname{cl}(x \cap y) \neq \varnothing$ if and only if both $\operatorname{cl}(w) \cap \operatorname{cl}(x) \neq \varnothing$ and $\operatorname{cl}(w) \cap \operatorname{cl}(y) \neq \varnothing$, and hence (ii) must be the case. Therefore, $\mathcal{M}_{2}$ satisfies (FS') and $\left(\mathrm{FP}^{\prime}\right)$. Observe that $\mathcal{M}_{2}$ does not satisfy $\left(\mathrm{C}^{\prime}\right)$, for the union of all $w$ such that $\neg C(w, x)$ is the complement of $x$, but it will contact $x$. This implies that $\mathcal{M}_{2}$ does not satisfy $\left(U^{\prime}\right)$ either.

Note that from the proof of Lemma 3.1, we can see that $\mathcal{M}_{2}$ can be defined in $\mathcal{M}_{1}$, for the set of nonempty regular open subsets of $R^{2}$ can be defined in $\mathcal{M}_{1}$ by $x=i(c(x))$. This fact will be utilized from time to time in the following. Next we will show that both $\mathcal{M}_{1}$ and $\mathcal{M}_{2}$ are strongly undecidable. A conceivable strategy for showing the strong undecidability of a structure $\mathcal{M}$ is to try to interpret the first-order language of arithmetic (it is sufficient to consider the language whose signature contains only two binary function symbols " + " and " $\times$ ") into the theory of $\mathcal{M}$ and then use that interpretation $I$ to define inside $\mathcal{M}$ a model $\mathcal{M}^{I}$ such that $\mathcal{M}^{I}$ is isomorphic to the intended model $(\omega ;+, \times)$ of the first-order arithmetic (for the reason why $\mathcal{M}$ will be strongly undecidable then, see Shoenfield [11, p. 136]). My first idea was also to adopt the aforementioned strategy. However, the traditional way will not succeed, ${ }^{9}$ for in order to follow that way, we have to come up first with a formula with a free variable which defines a countable infinite subset of $\operatorname{Dom}\left(\mathcal{M}_{1}\right)$ or $\operatorname{Dom}\left(\mathcal{M}_{2}\right)\left(\mathcal{M}_{1}\right.$ and $\mathcal{M}_{2}$ are as defined above) and then such a subset will serve as $\omega$, but this is impossible since as the following lemma shows, any definable nonempty subset of $\operatorname{Dom}\left(\mathcal{M}_{1}\right)$ or $\operatorname{Dom}\left(\mathcal{M}_{2}\right)$ will either be the set whose only member is $R^{2}$ or be uncountable.

Lemma 3.2 Consider mereotopological structures $\mathcal{M}_{1}$ and $\mathcal{M}_{2}$ as defined above. Any definable nonempty subset of $\operatorname{Dom}\left(\mathcal{M}_{1}\right)$ or $\operatorname{Dom}\left(\mathcal{M}_{2}\right)$ must either be the set whose only member is $R^{2}$ or be uncountable.

Proof It suffices to show the case of $\mathcal{M}_{1}$, for as mentioned above, $\mathcal{M}_{2}$ can be defined in $\mathcal{M}_{1}$. Suppose that $S \subseteq \operatorname{Dom}\left(\mathcal{M}_{1}\right)$ is nonempty, definable, and is not the 
set whose only member is $R^{2}$. Observe that $S^{\prime}=\left\{x \in \operatorname{Dom}\left(\mathcal{M}_{1}\right): \sim_{x} \in S\right\}$ is also definable, and obviously $S$ and $S^{\prime}$ have the same cardinality. Also observe that any homeomorphism $f$ on $R^{2}$ will determine an automorphism $\mathrm{F}_{f}$ on $\mathcal{M}_{1}$ in the following way: for any $u \in \operatorname{Dom}\left(\mathcal{M}_{1}\right)$, define $\mathrm{F}_{f}(u)=\left\{(x, y) \in R^{2}: \exists\left(x^{\prime}, y^{\prime}\right) \in\right.$ $\left.u f\left(x^{\prime}, y^{\prime}\right)=(x, y)\right\}$ (i.e., $\mathrm{F}_{f}(u)$ is the image of $u$ under $f$; it is easy to check that $\mathrm{F}_{f}$ is an automorphism on $\left.\mathcal{M}_{1}\right)$. Furthermore, it is known that if the signature of a language $L$ contains only finitely many predicates but no function symbols, then any first-order definable relation on an $L$-structure must be Gaifman local (see Libkin [7, Chapter 4]) . Now assume that the Gaifman locality ranks of $S$ and $S^{\prime}$ are $d$ and $d^{\prime}$, respectively. Consider the following two possibilities.

(a) There is some $s \in S$ such that $s$ or $\sim_{s}$ has interior. Assume first that $\sim_{s}$ has interior. Let $w$ be a closed disk which is included in $\sim_{s}$. Choose two distinct points from the boundary of $w$ such that $s$ intersects the line determined by these two points (this can be done since ${ }_{S} \neq R^{2}$ ). Now consider the segment $E$ determined by the said two points. Obviously, any point on $E$ is not in $s$. Now consider a function $f$ which moves every point in $R^{2}$ up along the slope of $E$ for a positive distance $r$ which is smaller than the length of $E$. It should be clear that $f$ is a homeomorphism on $R^{2}$. Moreover, as mentioned above, the function $\mathrm{F}_{f}$ defined as follows will be an automorphism on $\mathcal{M}_{1}$ : for any $u \in \operatorname{Dom}\left(\mathcal{M}_{1}\right)$, let $\mathrm{F}_{f}(u)=$ the image of $u$ under $f$. Since $S$ is Gaifman local with a rank $d$, for any $u \in \operatorname{Dom}\left(\mathcal{M}_{1}\right)$, if $N_{d}(u)$ is isomorphic to $N_{d}(s)$ with an isomorphism which maps $u$ to $s$, then $u \in S$ too, where $N_{d}(x)$ is the neighborhood of $x$ within the distance $d$, that is, $N_{d}(x)$ is the substructure of $\mathcal{M}_{1}$ such that $\operatorname{Dom}\left(N_{d}(x)\right)=\left\{y \in \operatorname{Dom}\left(\mathcal{M}_{1}\right)\right.$ : the distance between $x$ and $\left.y \leq d\right\}$ (again, see [7] for the definition of "distance"). By the definition just mentioned, $N_{d}(s)$ is a substructure of $\mathcal{M}_{1}$. Then let $\mathcal{M}^{\prime}$ be the substructure of $\mathcal{M}_{1}$ such that $\operatorname{Dom}\left(\mathcal{M}^{\prime}\right)=\left\{\mathrm{F}_{f}(u): u \in \operatorname{Dom}\left(N_{d}(s)\right)\right\}$. Since $\mathrm{F}_{f}$ is an automorphism on $\mathcal{M}_{1}, \mathcal{M}^{\prime}$ will be isomorphic to $N_{d}(s)$, but $\mathcal{M}^{\prime}$ is exactly $N_{d}\left(\mathrm{~F}_{f}(s)\right)$, and hence $\mathrm{F}_{f}(s) \in S$ too. However, there must be uncountably many $f$ 's such that $\mathrm{F}_{f}(s)$ 's are pairwise distinct, for there are uncountably many positive real numbers each of which is smaller than the length of $E$. This means that $S$ must be uncountable. If $\sim_{s}$ has no interior but $s$ has interior, we can apply the same argument as above to show that $S^{\prime}$ is uncountable (except that the Gaifman locality rank involved in this case will be $d^{\prime}$ ). But as mentioned earlier, $S^{\prime}$ and $S$ have the same cardinality, so $S$ will be uncountable too.

(b) For any $s \in S$, neither $s$ nor ${ }_{S}$ has interior, that is, both $s$ and ${ }_{s}$ are dense. Assume without loss of generality that $R^{2} \notin S$ (if $R^{2} \in S$, consider instead the set defined in $\mathcal{M}_{1}$ by the formula $\alpha(x) \wedge \neg \forall y P y x$, where $\alpha(x)$ defines $S$ in $\left.\mathcal{M}_{1}\right)$. If $S$ is countable, then there are two possibilities. (b.1) $S$ is finite, that is, for some $n, S=\left\{s_{0}, s_{1}, \ldots, s_{n}\right\}$, where for each $i, j \leq n, s_{i} \subset R^{2}$ and $s_{i} \neq s_{j}$ if $i \neq j$. Choose $n+1$ mutually disjoint closed disks $D_{0}, D_{1}, \ldots, D_{n}$ such that each $D_{i}$ contains a point $a_{i} \in s_{0}$ and a point $b_{i} \in \sim_{s_{i}}$ (this can be done since each $s_{i}$ and its complement are dense). Then it is easy to define a homeomorphism $f$ on $R^{2}$ such that $f\left(a_{i}\right)=b_{i}$ for $i \leq n$ and that $f(x)=x$ if $x \notin D_{0} \cup D_{1} \cup \cdots \cup D_{n}$. As mentioned above, $\mathrm{F}_{f}$ will be an automorphism on $\mathcal{M}_{1}$, and by the same argument as that in (a), $\mathrm{F}_{f}\left(s_{0}\right) \in S$. However, for any $i \leq n, \mathrm{~F}_{f}\left(s_{0}\right) \neq s_{i}$ since $f\left(a_{i}\right) \notin s_{i}$; therefore $\mathrm{F}_{f}\left(s_{0}\right) \notin S$, and we have a contradiction. (b.2) $S$ is infinite, that is, $S=\left\{s_{i} \subset R^{2}: i \in \omega\right.$ and $s_{i} \neq s_{j}$ if $\left.i \neq j\right\}$. Similar to the first case, we can choose $\omega$-many mutually disjoint closed disks $D_{0}, D_{1}, D_{2}, \ldots$ such that each 
$D_{i}$ contains a point $a_{i} \in s_{0}$ and a point $b_{i} \in \sim_{s_{i}}$ (again, this can be done since each $s_{i}$ and its complement are dense) and then define a homeomorphism $f$ on $R^{2}$ such that $f\left(a_{i}\right)=b_{i}$ for $i \in \omega$ and that $f(x)=x$ if $x \notin \bigcup_{i \in \omega} D_{i}$. Then for the same reason as above, $\mathrm{F}_{f}\left(s_{0}\right) \in S$ but $\mathrm{F}_{f}\left(s_{0}\right) \neq s_{i}$, for any $i \in \omega$, which is a contradiction. This shows that $S$ must be uncountable.

Owing to Lemma 3.2, the traditional way of defining a structure in another structure cannot work in our case. Nonetheless, thanks to the richness of $\operatorname{Dom}\left(\mathcal{M}_{2}\right)$, we can define a subset $S$ of $\operatorname{Dom}\left(\mathcal{M}_{2}\right)$ such that each member of $S$ can stand for $\omega$, and then we can give an interpretation $I$ relativized to members of $S$ such that every sentence $\alpha$ in the first-order language of arithmetic can be effectively translated into a sentence $\alpha^{I}$ in the first-order language of mereotopology, and that $(\omega,+, \times) \models \alpha$ if and only if $\mathcal{M}_{2} \models \alpha^{I}$, from which it will also follow that $(\omega,+, \times) \models \alpha$ if and only if $\mathcal{M}_{1} \models \forall x\left(x=i(c(x)) \rightarrow \alpha^{I}\right)$, for as mentioned earlier, the set of regular open subsets of $R^{2}$ is definable in $\mathcal{M}_{1}$ by the formula $x=i(c(x))$. Then it can be shown that $\mathcal{M}_{1}$ and $\mathcal{M}_{2}$ are strongly undecidable. Let us give a sketch as follows. Consider $\mathcal{M}_{2}$ first. If a mereotopological theory $T$ is satisfied by $\mathcal{M}_{2}, T$ will have a finite extension $T^{\prime}$ which proves $\exists x \varphi(x)$, where $\varphi(x)$ defines $S$ in $\mathcal{M}_{2}$, and then $T^{\prime}$ cannot be decidable, for otherwise there will be a decidable arithmetic theory that is satisfied by $(\omega,+, \times)$, which is impossible since $(\omega,+, \times)$ is strongly undecidable (see [11, p. 134] ). The same argument can also apply to any theory which is satisfied by $\mathcal{M}_{1}$, for again, $\mathcal{M}_{2}$ can be defined in $\mathcal{M}_{1}$. The situation here is kind of interesting: we cannot define in $\mathcal{M}_{1}$ or in $\mathcal{M}_{2}$ a structure which is isomorphic to $(\omega,+, \times)$ since the set of natural numbers is not definable in $\mathcal{M}_{1}$ or in $\mathcal{M}_{2}$, but we can find in $\mathcal{M}_{1}$ or in $\mathcal{M}_{2}$ some objects each of which is, roughly speaking, isomorphic to $(\omega,+, \times)$ and, in fact, there are uncountably many objects of such a kind in $\mathcal{M}_{1}$ or in $\mathcal{M}_{2}$. Even though we cannot pick just one object of the said kind and then define it formally, as we will see, for our purpose this does not matter.

We give a detailed proof now. As mentioned in the foregoing remarks, it suffices to deal with the case of $\mathcal{M}_{2}$ and then the case of $\mathcal{M}_{1}$ will follow immediately. More precisely, we will show each of the following propositions.

(1) We can define in $\mathcal{M}_{2}$ a class of objects which will be named "chains." See Figure 1 for an intuitive view of what a chain looks like. ${ }^{10}$

(2) We can define in $\mathcal{M}_{2}$ a binary relation such that two chains (which do not contact each other) stand in this relation if and only if they are equinumerous, that is, they have the same number of components (a component of a chain is a maximally connected part of that chain; see below).

(3) We can define in $\mathcal{M}_{2}$ the class of finite chains (a finite chain has only finitely many components).

(4) We can define in $\mathcal{M}_{2}$ a class of objects which will be named "UFC" (union of finite chains). A UFC is the union of a collection of finite chains in which any

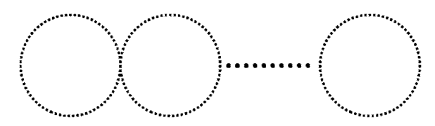

Figure 1 A chain. 
two distinct chains are not equinumerous to each other and in which any chain is equinumerous to a chain with one component removed. Intuitively, the set of natural numbers can be represented by a UFC.

(5) We can define addition and multiplication in a UFC. They will be four-place relations in $\mathcal{M}_{2}$. For example, $x+y=z$ in $\mathrm{U}$ iff $\mathrm{U}$ is a UFC; $x, y$, and $z$ are chains in $\mathrm{U}$; and $z$ is equinumerous to the chain generated by concatenating $x$ and $y$ (this is the idea but the exact definition will be more circuitous).

(6) Based on the definitions given in (5), we can effectively translate each formula in the first-order language of arithmetic into a formula in the first-order language of mereotopology.

(7) We can show that any sentence in the first-order language of arithmetic is true in $(\omega,+, \times)$ if and only if its translation is true in $\mathcal{M}_{2}$ and from this we can show that $\mathcal{M}_{2}$ is strongly undecidable.

Proof of (1) First observe that we can define "self-connectedness" as follows:

$$
\begin{gathered}
\mathrm{SC}(x)=_{\mathrm{df}} \quad \forall y \forall z((\neg O(y, z) \wedge x=y+z) \rightarrow \exists u \exists t(P(u, y) \wedge P(t, z) \\
\left.\left.\wedge C(u, t) \wedge \neg C\left(u+t, \sim{ }^{\prime}\right)\right)\right) .{ }^{11}
\end{gathered}
$$

Then we can define " $x$ is a maximally-connected part of $y$ " as follows:

$\mathrm{MP}(x, y)=_{\mathrm{df}} \mathrm{SC}(x) \wedge(x=y \vee(P P(x, y) \wedge \neg \exists z(P(z, y) \wedge \mathrm{SC}(z) \wedge P P(x, z))))$.

Next we will define "chain," and in order to make the definition more readable, it will first be expressed in English, and then be translated into a formula in the first-order language of mereotopology.

We have Chain $(x)$ iff $x$ is self-connected or $x$ has at least two maximally connected parts, and there are exactly two of them each of which contacts, but not overlaps, exactly one maximally connected part of $x$, and each maximally connected part of $x$ except the said two contacts but not overlaps exactly two maximally connected parts of $x$.

Formally,

$$
\begin{array}{rl}
\text { Chain }(x)=_{\mathrm{df}} & \mathrm{SC}(x) \vee \exists y \exists z(y \neq z \wedge \mathrm{MP}(y, x) \wedge \mathrm{MP}(z, x) \wedge \exists ! u(\operatorname{MP}(u, x) \\
& \wedge u \neq y \wedge C(u, y)) \wedge \exists ! u(\operatorname{MP}(u, x) \wedge u \neq z \wedge C(u, z)) \\
& \wedge \forall v(\exists ! u(\operatorname{MP}(u, x) \wedge u \neq v \wedge C(u, v)) \rightarrow(v=y \vee v=z)) \\
& \wedge \forall v((\operatorname{MP}(v, x) \wedge v \neq y \wedge v \neq z) \rightarrow \exists u \exists t((\operatorname{MP}(u, x) \\
& \wedge \operatorname{MP}(t, x) \wedge u \neq v \wedge t \neq v \wedge \neg C(u, t) \wedge C(v, u) \wedge C(v, t)) \\
& \wedge \forall z((\operatorname{MP}(z, x) \wedge z \neq v \wedge C(z, v)) \rightarrow(z=u \wedge z=t))))) .
\end{array}
$$

Proof of (2) We will give a general definition of the equinumerosity between two disjoint collections each of which consists of some maximally connected parts. Again, we first give an informal version and then a formal one.

We have $\mathrm{EC}(x, y)$ iff $x=y$, or $x$ and $y$ do not contact each other and there is some $z$ which is composed of $x$ and $y$ as well as some maximally connected parts which neither overlap $x$ nor overlap $y$ such that for each maximally connected part 
$u$ of $x$ there is exactly one maximally connected part $v$ of $y$ such that $u$ and $v$ are connected via exactly one maximally connected part of $z$, and for each maximally connected part $u$ of $y$ there is exactly one maximally connected part $v$ of $x$ such that $u$ and $v$ are connected via exactly one maximally connected part of $z$.

Formally,

$$
\begin{aligned}
\mathrm{EC}(x, y)=_{\mathrm{df}} & =y \vee(\neg C(x, y) \wedge \exists z(P P(x, z) \wedge P P(y, z) \wedge \forall u(\operatorname{MP}(u, x) \\
& \rightarrow \mathrm{MP}(u, z)) \wedge \forall u(\operatorname{MP}(u, y) \rightarrow \operatorname{MP}(u, z)) \wedge \forall u(\operatorname{MP}(u, x) \\
& \rightarrow \exists ! t(\operatorname{MP}(t, y) \wedge \exists ! v(\operatorname{MP}(v, z) \wedge \neg O(v, x) \wedge \neg O(v, y) \\
& \wedge C(v, u) \wedge C(v, t) \wedge \forall w((\operatorname{MP}(w, z) \wedge w \neq u \wedge w \neq t \wedge w \neq v) \\
& \rightarrow \neg C(v, w))))) \wedge \forall u(\operatorname{MP}(u, y) \rightarrow \exists ! t(\operatorname{MP}(t, x) \wedge \exists ! v(\operatorname{MP}(v, z) \\
& \wedge \neg O(v, x) \wedge \neg O(v, y) \wedge C(v, u) \wedge C(v, t) \wedge \forall w((\operatorname{MP}(w, z) \\
& \wedge w \neq u \wedge w \neq t \wedge w \neq v) \rightarrow \neg C(v, w))))))) .
\end{aligned}
$$

Proof of (3) We define a class of chains which will be called "even." Intuitively, a chain $x$ is even if and only if each component $u$ of $x$ can be paired with exactly one component $v$ of $x$, where $u \neq v$. This motivates the following definition:

$$
\begin{aligned}
\operatorname{Even}(x)={ }_{\mathrm{df}} & \operatorname{Chain}(x) \wedge \exists y \exists z(y \neq z \wedge \mathrm{MP}(y, x) \wedge \mathrm{MP}(z, x)) \wedge \exists y(P P(y, x) \\
& \wedge \forall z(\operatorname{MP}(z, x) \rightarrow \exists ! u(\operatorname{MP}(u, y) \wedge P P(u, z))) \wedge \forall u(\operatorname{MP}(u, y) \\
& \rightarrow \exists ! v(\operatorname{MP}(v, y) \wedge \neg O(u, v) \wedge C(u, v)))) .
\end{aligned}
$$

Now a chain $x$ is finite if and only if it is not the case that both $x$ and the chain formed by removing one component from $x$ are even. Let FC stand for "finite chain."

Formally,

$$
\begin{array}{r}
\mathrm{FC}(x)=_{\mathrm{df}} \operatorname{Chain}(x) \wedge \neg(\operatorname{Even}(x) \wedge \exists y(\operatorname{Chain}(y) \wedge P P(y, x) \wedge \exists ! z(\mathrm{MP}(z, x) \\
\wedge \neg O(z, y) \wedge \forall u((\mathrm{MP}(u, x) \wedge u \neq z) \rightarrow P(u, y))) \wedge \operatorname{Even}(y))) .
\end{array}
$$

Proof of (4) Let us now define "union of finite chains" (UFC). First we give the informal version.

We have UFC $(x)$ iff $x$ is composed of some finite chains which do not contact each other, and $x$ contains a finite chain which is composed of exactly one maximally connected part (i.e., itself), and for any finite chain $y$ in $x$ there is another finite chain $z$ in $x$ such that $y$ is equinumerous to a proper subchain $u$ of $z$, where $u$ is formed by removing one maximally connected part of $z$, and for any two distinct finite chains $y$ and $z$ in $x$, one must be equinumerous to a proper subchain of the other, and for any maximally connected part $y$ of $x$, there is some finite chain $z$ of $x$ such that $y$ is a part of $z$ (the final clause is to make sure that $x$ is composed of finite chains only).

Before giving a formal version, let us define the following predicates:

$$
\begin{aligned}
\text { Proper Chain Part : } \mathrm{PC}(x, y) \\
={ }_{\mathrm{df}} P P(x, y) \wedge \mathrm{Chain}(x) \wedge \forall u(\mathrm{MP}(u, x) \rightarrow \mathrm{MP}(u, y)) ; \\
\text { Maximal Chain Part : } \mathrm{MC}(x, y) \\
={ }_{\mathrm{df}} \text { Chain }(x) \wedge P(x, y) \wedge \forall z(\operatorname{MP}(z, x) \rightarrow \mathrm{MP}(z, y)) \\
\quad \wedge \neg \exists z(P P(z, y) \wedge P P(x, z) \wedge \text { Chain }(z)) ;
\end{aligned}
$$




$$
\begin{aligned}
& \text { Successor : } \mathrm{S}(x, y) \\
& =_{\mathrm{df}} \text { Chain }(x) \wedge \text { Chain }(y) \wedge \neg C(x, y) \\
& \wedge \exists z(\mathrm{PC}(z, x) \wedge \exists ! u(\mathrm{MP}(u, x) \wedge \neg O(u, z)) \wedge \mathrm{EC}(z, y)) \\
& \operatorname{UFC}(x)=_{\mathrm{df}} \forall y(\operatorname{MC}(y, x) \rightarrow \mathrm{FC}(y)) \wedge \forall y \forall z((\operatorname{MC}(y, x) \wedge \operatorname{MC}(z, x) \wedge y \neq z) \\
& \rightarrow \neg C(y, z)) \wedge \exists y(\mathrm{MC}(y, x) \wedge \mathrm{SC}(y)) \wedge \forall y(\mathrm{MC}(y, x) \\
& \rightarrow \exists z(\mathrm{MC}(z, x) \wedge S(z, y))) \wedge \forall y \forall z((\operatorname{MC}(y, x) \wedge \mathrm{MC}(z, x) \\
& \wedge y \neq z) \rightarrow \exists u((\mathrm{PC}(u, z) \wedge \mathrm{EC}(u, y)) \vee(\mathrm{PC}(u, y) \\
& \wedge \mathrm{EC}(u, z)))) \wedge \forall y(\operatorname{MP}(y, x) \rightarrow \exists z(\operatorname{MC}(z, x) \wedge P(y, z))) .
\end{aligned}
$$

It should be clear that in $\mathcal{M}_{2}$ there is some member which satisfies the condition for "union of finite chains," so UFC $(x)$ defines a nonempty subset of $\operatorname{Dom}\left(\mathcal{M}_{2}\right){ }^{12}$

Proof of (5) We define next a four-place predicate $\operatorname{Add}(u, x, y, z)$ which expresses the following conditions: (i) $u$ is a UFC; (ii) $x, y$, and $z$ are finite chains in $u$; (iii) if $x$ consists of only one component, $z=y$, and if $y$ consists of only one component, $z=x$; (iv) if neither $x$ nor $y$ consists of only one component, then the predecessor of $x$ is equinumerous to a proper subchain $w$ of $z, y$ is equinumerous to a proper subchain $v$ of $z, w$ and $v$ are disjoint, and $z$ is composed of $w$ and $v$. Now let $0^{u}$ be the finite chain of $u$ which consists of only one component. Then formally,

$$
\begin{aligned}
\operatorname{Add}(u, x, y, z)=_{\mathrm{df}} & \operatorname{UFC}(u) \wedge \mathrm{MC}(x, u) \wedge \mathrm{MC}(y, u) \wedge \mathrm{MC}(z, u) \\
& \wedge\left(\left(x=0^{u} \rightarrow z=y\right) \wedge\left(y=0^{u} \rightarrow z=x\right)\right) \\
& \wedge\left(\left(x \neq 0^{u} \wedge y \neq 0^{u}\right) \rightarrow \exists t \exists w \exists v(\mathrm{MC}(t, u) \wedge \mathrm{S}(x, t)\right. \\
& \wedge \mathrm{PC}(w, z) \wedge \mathrm{PC}(v, z) \wedge \neg O(w, v) \\
& \wedge z=w+v \wedge \mathrm{EC}(w, t) \wedge \mathrm{EC}(y, v))) .
\end{aligned}
$$

Note that $\mathcal{M}_{2}$ is a model of GEMT and hence + is well defined in $\mathcal{M}_{2}$. Also note that since $0^{u}$ is the chain with one component, the interpretation of a natural number $n$ will be a finite chain which has $n+1$ components and hence the interpretation of $n+m$ will be equinumerous to the concatenation of the interpretations of $n-1$ and $m$ (if $n \neq 0$ ).

Similar to the case of addition, the interpretation of multiplication will also be a four-place predicate $\operatorname{Mul}(u, x, y, z)$ which expresses the following conditions: (i) $u$ is a UFC; (ii) $x, y$, and $z$ are finite chains in $u$; (iii) if $x=0^{u}$ or $y=0^{u}$, then $z=0^{u}$; (iv) if $x$ consists of exactly two components, $z=y$, and if $y$ consists of exactly two components, $z=x$; (v) otherwise, there is some $w$ which is equinumerous to the predecessor of $z$ and which is composed of some maximal chain parts each of which is equinumerous to the predecessor of $x$, and there is some $w^{\prime}$ which has $w$ and the predecessor of $y$ as proper parts such that each component of the predecessor of $y$ is linked via some maximally connected part of $w^{\prime}$ with exactly one maximal chain part of $w$, and conversely, each maximal chain part of $w$ is linked via some maximally connected part of $w^{\prime}$ with exactly one component of the predecessor of $y$. Now let $1^{u}$ be 
the finite chain of $u$ which is composed of exactly two components. Then formally,

$$
\begin{array}{rl}
\operatorname{Mul}(u, x, y, z)={ }_{\mathrm{df}} & \mathrm{UFC}(u) \wedge \mathrm{MC}(x, u) \wedge \mathrm{MC}(y, u) \wedge \mathrm{MC}(z, u) \\
& \wedge\left(\left(x=0^{u} \vee y=0^{u}\right) \rightarrow z=0^{u}\right) \wedge\left(\left(x=1^{u} \rightarrow z=y\right)\right. \\
& \left.\wedge\left(y=1^{u} \rightarrow z=x\right)\right) \wedge\left(\left(x \neq 0^{u} \wedge x \neq 1^{u} \wedge y \neq 0^{u}\right.\right. \\
& \left.\wedge y \neq 1^{u}\right) \rightarrow \exists x^{\prime} \exists y^{\prime} \exists z^{\prime}\left(\mathrm{MC}\left(x^{\prime}, u\right) \wedge \mathrm{MC}\left(y^{\prime}, u\right) \wedge \mathrm{MC}\left(z^{\prime}, u\right)\right. \\
& \wedge \mathrm{S}\left(x, x^{\prime}\right) \wedge \mathrm{S}\left(y, y^{\prime}\right) \wedge \mathrm{S}\left(z, z^{\prime}\right) \wedge \exists w\left(\mathrm{EC}\left(w, z^{\prime}\right)\right. \\
& \wedge \exists z \mathrm{MC}(z, w) \wedge \forall y \forall z((\operatorname{MC}(y, w) \wedge \operatorname{MC}(z, w) \wedge y \neq z) \\
& \rightarrow \neg C(y, z)) \wedge \forall z\left(\operatorname{MC}(z, w) \rightarrow \operatorname{EC}\left(z, x^{\prime}\right)\right) \\
& \wedge \exists w^{\prime}\left(P P ( w , w ^ { \prime } ) \wedge P P ( y ^ { \prime } , w ^ { \prime } ) \wedge \forall u \left(\left(\operatorname{MP}\left(u, y^{\prime}\right)\right.\right.\right. \\
& \left.\left.\rightarrow \mathrm{MP}\left(u, w^{\prime}\right)\right) \wedge\left(\operatorname{MP}(u, w) \rightarrow \operatorname{MP}\left(u, w^{\prime}\right)\right)\right) \\
& \wedge \forall u\left(\operatorname { M P } ( u , y ^ { \prime } ) \rightarrow \exists ! z \left(\operatorname { M C } ( z , w ) \wedge \exists t \left(\operatorname{MP}\left(t, w^{\prime}\right)\right.\right.\right. \\
& \wedge \neg O(u, t) \wedge \neg O(z, t) \wedge C(u, t) \wedge C(z, t)))) \\
& \wedge \forall z\left(\operatorname { M C } ( z , w ) \rightarrow \exists ! u \left(\operatorname { M P } ( u , y ^ { \prime } ) \wedge \exists t \left(\operatorname{MP}\left(t, w^{\prime}\right)\right.\right.\right. \\
& \wedge \neg O(u, t) \wedge \neg O(z, t) \wedge C(u, t) \wedge C(z, t))))))) .
\end{array}
$$

Proof of (6) Let $\mathrm{N}(u)$ be an abbreviation of

$$
\begin{aligned}
& \operatorname{UFC}(u) \wedge \forall y \forall z((\operatorname{MC}(x, u) \wedge \operatorname{MC}(y, u)) \\
& \left.\quad \rightarrow \exists ! z \exists ! z^{\prime}\left(\operatorname{MC}(z, u) \wedge \operatorname{MC}\left(z^{\prime}, u\right) \wedge \operatorname{Add}(u, x, y, z) \wedge \operatorname{Mul}\left(u, x, y, z^{\prime}\right)\right)\right) .
\end{aligned}
$$

It is easy to see that $\mathrm{N}(u)$ defines in $\mathcal{M}_{2}$ a nonempty set (see the example given in endnote 12) and, intuitively, each $u$ in such a set will be isomorphic to $(\omega,+, \times)$ if we treat " + " and " $x$ " as predicates which denote the graphs of the original functions. Now every first-order arithmetic formula $\alpha$ (in the language which treats "+" and " $x$ " as predicates) can be effectively translated in the following two-step way into a formula in the language of mereotopology.

First step: Translate each " $\forall x$ " in $\alpha$ into " $\forall x(\mathrm{MC}(x, u) \rightarrow$," each " $\exists x$ " in $\alpha$ into " $\exists x(\operatorname{MC}(x, u) \wedge, "$ and replace each $+(x, y, z)$ and $\times(x, y, z)$ with $\operatorname{Add}(u, x, y, z)$ and $\operatorname{Mul}(u, x, y, z)$. Call the resultant formula $\alpha^{*}$.

Second step: If there is no free variable in $\alpha$, the final translation $\alpha^{T}$ will be $\forall u\left(\mathrm{~N}(u) \rightarrow \alpha^{*}\right)$. Otherwise, suppose that there are $n$ free variables $x_{1}, \ldots, x_{n}$ in $\alpha$. Then the final translation $\alpha^{T}$ will be $\forall u\left(\left(\mathrm{~N}(u) \wedge \mathrm{MC}\left(x_{1}, u\right) \wedge \cdots \wedge \mathrm{MC}\left(x_{n}, u\right)\right)\right.$ $\left.\rightarrow \alpha^{*}\right)$.

It should be clear that the foregoing procedure is effective.

Proof of (7) As mentioned above, $\mathrm{N}(u)$ defines in $\mathcal{M}_{2}$ a nonempty set and each $u$ in that set is isomorphic to $(\omega,+, \times)$. Naturally, the isomorphism involved will be from natural numbers in $\omega$ to finite chains in $u$ such that 0 is mapped to the chain composed of one component, 1 to the chain composed of two components, and so on. In this light, we can see that for any first-order arithmetic sentence $\alpha,(\omega,+, \times) \models \alpha$ if and only if $\mathcal{M}_{2} \models \alpha^{T}$.

Now we will show that $\mathcal{M}_{2}$ is strongly undecidable, which will utilize the fact that $(\omega,+, \times)$ is strongly undecidable. Consider any mereotopological theory $T$ which is satisfied by $\mathcal{M}_{2}$. Suppose that $T$ is decidable. Consider the following set 
of first-order arithmetic sentences $H=\left\{\alpha: T \models \alpha^{T}\right\}$. Since $\mathcal{M}_{2} \models T$, for each $\alpha \in H, \mathcal{M}_{2} \models \alpha^{T}$ and hence $(\omega,+, \times) \models \alpha$. $H$ is decidable, for $T$ is decidable and the translation from $\alpha$ to $\alpha^{T}$ is effective. Next we will show that $H$ is a logical theory, that is, $H$ is closed under logical implication. For any $\gamma$ such that $H \models \gamma$, by compactness, there are some $\alpha_{1}, \ldots, \alpha_{n} \in H$ such that $\alpha_{1}, \ldots, \alpha_{n} \models \gamma$. Therefore, $\left(\alpha_{1} \wedge \cdots \wedge \alpha_{n}\right) \rightarrow \gamma$ is a logical truth and it follows that $T \models\left(\left(\alpha_{1} \wedge \cdots \wedge \alpha_{n}\right) \rightarrow \gamma\right)^{T}$, that is, (i) $T \models \forall u\left(\mathrm{~N}(u) \rightarrow\left(\left(\alpha_{1}^{*} \wedge \cdots \wedge \alpha_{n}^{*}\right) \rightarrow \gamma^{*}\right)\right)$. Besides, we also know that (ii) for each $1 \leq i \leq n, T \models \alpha_{i}^{T}$, that is, $T \models \forall u\left(\mathrm{~N}(u) \rightarrow \alpha_{i}^{*}\right)$. From (i) and (ii), we have $T \models \forall u\left(\mathrm{~N}(u) \rightarrow \gamma^{*}\right)$, that is, $T \models \gamma^{T}$. So $\gamma \in H$ too, and this shows that $H$ is a logical theory. However, $H$ is satisfied by $(\omega,+, \times)$ and then by the strong undecidability of $(\omega,+, \times), H$ is undecidable and hence we have a contradiction. Therefore, $T$ cannot be decidable, which shows that $\mathcal{M}_{2}$ is strongly undecidable.

Since $\mathcal{M}_{2}$ is definable in $\mathcal{M}_{1}$, it follows that $\mathcal{M}_{1}$ is strongly undecidable too (again, see [11, p. 134]).

\section{Concluding Remarks}

So all mereotopological theories except those of the form $\mathbf{S A X}$ or $\mathbf{S A X} \mathbf{X}^{\prime}$ or $\mathbf{S B} \mathbf{X}^{\prime}$, where $\mathbf{X}$ is strictly stronger than CEMT, are undecidable (my guess is that any theory of any of the aforementioned forms is also undecidable). Moreover, from the results given above, we can also get information about other kinds of undecidability. Let us introduce the following definitions first.

Definition 4.1 A theory $T$ is essentially undecidable if and only if all its consistent extensions in the same language are undecidable.

Definition 4.2 A theory $T$ is hereditarily undecidable if and only if all its subtheories are undecidable.

Definition 4.3 A theory $T$ is strongly undecidable if and only if any theory in the same language which is compatible with $T$ is undecidable. ${ }^{13}$

Obviously, all mereotopological theories are neither essentially undecidable nor strongly undecidable, for from the proof of separability, we can see that they have decidable consistent extensions in the same language. Moreover, all mereotopological theories except those of any of the forms mentioned above are hereditarily undecidable, for they are either finitely inseparable or are satisfied by some strongly undecidable models.

The proof which shows the strong undecidability of $\mathcal{M}_{1}$ and $\mathcal{M}_{2}$ also shows that $\operatorname{Th}\left(\mathcal{M}_{1}\right)$ and $\operatorname{Th}\left(\mathcal{M}_{2}\right)$ cannot be arithmetical. Otherwise, since the procedure for translating $\alpha$ into $\alpha^{T}$ is effective and $(\omega,+, \times) \models \alpha$ if and only if $\mathcal{M}_{2} \models \alpha^{T}$, Th $((\omega,+, \times))$ will be arithmetical too, which is impossible owing to Tarski's undefinability theorem (see Enderton [5, p. 236]). Furthermore, $\operatorname{Th}\left(\mathcal{M}_{1}\right)$ and $\operatorname{Th}\left(\mathcal{M}_{2}\right)$ are at least as strong as the second-order arithmetic in the following sense. If $u$ satisfies $\mathrm{N}(u)$ in $\mathcal{M}_{2}$, the power set of chains in $u$ can be defined by $\operatorname{Sub}(x, u)={ }_{\mathrm{df}} P(x, u) \wedge \forall y((\operatorname{MP}(y, x) \rightarrow \mathrm{MP}(y, u)) \wedge(\mathrm{MC}(y, x) \rightarrow \mathrm{MC}(y, u)))$; that is, each $x$ which satisfies $\operatorname{Sub}(x, u)$ is the union of some chains in $u$, so $x$ can stand for a subset of $\omega$. In this light, each $u$ which satisfies $\mathrm{N}(u)$ in $\mathcal{M}_{2}$ will in effect 
be isomorphic to the intended two-sorted structure $(\omega, P(\omega),+, \times)$ of second-order arithmetic.

Some readers might think that intuitively both the power set of $R^{2}$ and the set of regular open subsets of $R^{2}$ are very rich and hence it is not surprising at all that we can "do arithmetic" on such domains. However, even though they are indeed very rich, we cannot describe the way we do arithmetic on them if the formal language (with an intended interpretation) is not expressive enough. For example, if the language contains only one predicate $P$ whose interpretation is the set inclusion, it is impossible to interpret the first-order arithmetic into the power set of $R^{2}$ or into the set of regular open subsets of $R^{2}$, for the mereological structure $\mathcal{M}$ based on either domain can only be a Boolean algebra with the least member removed and it is known that the theory of the said $\mathcal{M}$ is decidable (see [16]). But as we have seen here, with an additional predicate $C$ and a suitable intended interpretation, the expressiveness of the language increases amazingly. So language matters.

Finally, an anonymous referee brings to my attention the decidability issue of complete Boolean algebras with a relation of "overlapping." Here I will make some quick observations. Let " $\mathrm{O}$ *" stand for the relation of "overlapping." Then any complete Boolean algebra with such a relation must satisfy the following conditions (see Ciraulo [2]):

$$
\begin{aligned}
& \left(\mathrm{O}^{*} 1\right) O^{*}(x, y) \rightarrow O^{*}(y, x) \text { (symmetry); } \\
& \text { (O*2) } O^{*}(x, y) \rightarrow O^{*}(x,(x \wedge y)) \text { (meet closure); } \\
& \text { (O*3) } O^{*}\left(x, \bigvee_{i \in I} y_{i}\right) \text { iff } \exists_{i \in I} O^{*}\left(x, y_{i}\right) \text { (splitting of join); } \\
& \text { (O*4) } \forall z\left(O^{*}(z, x) \rightarrow O^{*}(z, y)\right) \rightarrow x \leq y \text { (density). }
\end{aligned}
$$

In order to make use of the results given in this paper or in previous ones, let us first translate the foregoing conditions into mereological axioms with "O*" as an additional primitive. $\left(\mathrm{O}^{*} 1\right)$ will remain intact. $\left(\mathrm{O}^{* 2}\right)$ will become " $O^{*}(x, y) \rightarrow O^{*}(x, x \times y)$ "; that is, we use "finite product" to stand for "meet." $(\mathrm{O} * 3)$ is not really first-order axiomatizable, but its first-order approximation may be defined by the following schema:

$$
\begin{aligned}
\exists x \alpha(x) \rightarrow & \exists z \forall y((O(y, z) \leftrightarrow \exists x(\alpha(x) \wedge O(y, x))) \\
\wedge & \left.\left(O^{*}(y, z) \leftrightarrow \exists x\left(\alpha(x) \wedge O^{*}(y, x)\right)\right)\right),
\end{aligned}
$$

for any formula $\alpha$ in which $x$ is free but $z$ and $y$ do not occur free ( $\alpha$ might have free variables other than $x$ ); that is, we use the fusion of the members of a definable (with or without parameters) subset $S$ of the domain to stand for the join of such members. $(\mathrm{O} * 4)$ will be " $\forall z\left(O^{*}(z, x) \rightarrow O^{*}(z, y)\right) \rightarrow P(x, y)$ "; that is, we use parthood relation to stand for the ordering.

If we interpret " $\mathrm{O} *$ " as the usual overlap relation, that is,

$$
\text { “ } O^{*}(x, y) \leftrightarrow \exists z(P(z, x) \wedge P(z, y)), "
$$

then $(\mathrm{O} * 1)-(\mathrm{O} * 4)$ will be theorems of GEM. However, it is known that GEM is decidable and hence the first-order approximation of the theory of complete Boolean algebras is decidable (see [16]). On the other hand, if we interpret " $\mathrm{O} *$ " as "contact," it is obvious that GEMT $+\left(\mathrm{O}^{*} 1\right)+\left(\mathrm{O}^{*} 2\right)+(\mathrm{O} * 4)$ is undecidable, for it is satisfied by $\mathcal{M}_{2}$. Nonetheless, neither $\mathcal{M}_{1}$ nor $\mathcal{M}_{2}$ can satisfy $(\mathrm{O} * 3)$. For example, for any regular open $x \neq R^{2}$, the fusion $\mathrm{z}$ of all $\mathrm{y}$ such that $\neg C(x, y)$ is the interior of the complement of $x$; however, no $y$ of the said kind can contact $x$ even though $z$ contacts $x$. It seems that any model of SGEMT' can satisfy $\left(\mathrm{O}^{*} 1\right)-(\mathrm{O} * 4)$ if "O*" is 
interpreted as "contact," for intuitively $\left(\mathrm{UF}^{\prime}\right)$ fits $\left(\mathrm{O}^{*} 3\right)$ well. However, the problem is that a model of SGEMT' is not necessarily a Boolean algebra with the least member removed. The foregoing are just some preliminary observations, and I do have some further questions in my mind now. However, I will not pursue this issue here but will probably try to address it in a much more detailed manner somewhere else.

\section{Notes}

1. The predicate "contact" is called "be connected to" by Casati and Varzi [1]. They have basically followed the nomenclature given by Whitehead [18]. However, as PrattHartmann has pointed out in $[10$, p. 22], such a term risks confusion with the standard topological notion of "connectedness." Hence I will follow the more recent usage and adopt the name "contact." By the way, as a matter of fact, the term "contact" instead of "be connected to" was first used by Düntsch and Orłowska [4], who also proved a result of undecidability. What they considered is so-called "contact relation algebra," abbreviated as CRA. A CRA is an algebra of binary relations on a given domain $U$, and such an algebra is generated from a relation $C$ on $U$, where $C$ satisfies $C(x, x), C(x, y) \rightarrow C(y, x)$ and $\forall z(C(x, z) \leftrightarrow C(y, z)) \rightarrow x=y$, by applying the following operations: union, intersection, complementation, composition $(R \mid Q=\{(x, y): \exists z(R(x, z) \wedge Q(z, y))\})$, and converse $\left(R^{\cup}=\{(x, y): R(y, x)\}\right)$. Düntsch and Orłowska have shown that the equational theory of CRA is undecidable. Let $L$ be a first-order language which has only one binary predicate " $C$," and consider any formula $\alpha$ of $L$ of the form $\psi \leftrightarrow \varphi$, where $\psi$ and $\varphi$ contain at most three variables, two of which are free ( $\psi$ and $\varphi$ must have the same free variables). Then the aforementioned result amounts to saying that the set $S$ of formulas of the said form, each of which can be proved from $C(x, x), C(x, y) \rightarrow C(y, x)$ and $\forall z(C(x, z) \leftrightarrow C(y, z)) \rightarrow x=y$, is undecidable (see [4, pp. 242-43]). If we define $P(x, y)={ }_{\mathrm{df}} \forall z(C(x, z) \rightarrow C(y, z)), P$ is obviously reflexive, transitive, and antisymmetric. Then such a result in effect says that the set of formulas of the aforementioned form, each of which can be proved from SGMT (see below for its definition), is undecidable. This implies that SGMT is undecidable, for we can effectively determine whether a formula is of that form or not. However, this fact does not imply that any meretopological theory strictly stronger than SGMT will also be undecidable. The undecidability of SGMT is indeed one of the results given in this paper (actually, the result given here is even stronger: SGMT is finitely inseparable; see Theorem 2.3), but this paper is only concerned with logical theories, where a logical theory is a set of sentences which is closed under logical implication, and hence whether a proper subset of a logical theory is decidable or not will not be considered here.

2. Casati and Varzi also mentioned a complicated axiom which is called "Whitehead's principle" (see [1, p. 61]): (W) $C(x, y) \rightarrow \exists z(S C(z) \wedge O(z, x) \wedge O(z, y) \wedge$ $\forall w(P(w, z) \rightarrow(O(w, x) \vee O(w, y))))$, where $S C(x)=_{\mathrm{df}} \forall y \forall z(\forall w(O(w, x) \leftrightarrow$ $(O(w, y) \vee O(w, z))) \rightarrow C(y, z))$. However, they refrained from endorsing such an axiom. Actually, (W) is hard to satisfy; it will fail in some reasonable domains, for example, $\operatorname{ROS}\left(R^{3}\right)$ (see [10, p. 19] for the definition of $\operatorname{ROS}\left(R^{n}\right)$ ). In this light, I will temporarily leave $(\mathrm{W})$ out.

3. Casati and Varzi's original definitions (including those of topological operators to be seen below) define only partial functions. Here I have modified the definitions in a harmless way so as to make each function total. 
4. Casati and Varzi did not consider $\mathbf{X T}+(\mathrm{K} 1)+(\mathrm{K} 2)+(\mathrm{K} 3)$ for any $\mathbf{X}$ which is strictly weaker than GEM.

5. This lemma comes from [8, p. 272], but the version presented here has been rephrased to make it more readable. By the way, I refer readers who would like to know more about how to interpret a language into a theory to Enderton [5, Section 2.7].

6. Note that in order to satisfy (SSP), there will be no least member in the model to be constructed and this is why the empty set has to be removed.

7. This is an easy theorem of general topology, but let us give a quick proof as follows. Consider $D=\mathcal{R O}\left(R^{2}\right) \backslash\{\emptyset\}$. Suppose that there are some $x, y \in D$ such that for all $z \in D, \operatorname{cl}(z) \cap \operatorname{cl}(x) \neq \varnothing$ implies that $\operatorname{cl}(z) \cap \operatorname{cl}(y) \neq \emptyset$, but $x$ is not a subset of $y$. If $x$ and $y$ are disjoint, then there will be a regular open nonempty proper subset $z$ of $x$ such that $\operatorname{cl}(z) \subset x$ (this is because $R^{2}$ is compact Hausdorff; see Munkres [9, p. 185]) and hence $\operatorname{cl}(z) \cap \operatorname{cl}(y)=\emptyset$, which contradicts the assumption. So $u=x \cap y \neq \emptyset$. Consider $\operatorname{cl}(x-u)$. Such a set must have a regular open interior, say, $v$, and again there will be a regular open nonempty proper subset $z$ of $v$ such that $\operatorname{cl}(z) \subset v$ and hence $\operatorname{cl}(z) \cap \operatorname{cl}(y)=\emptyset$, which again contradicts the assumption.

8. Strictly speaking, here " $\sim x$ " and " $x+y$ " should be " $\sim^{\mathcal{M}_{1}} x$ " and " $x+{ }^{\mathcal{M}_{1}} y$ ", respectively, where $\sim^{\mathcal{M}_{1}}$ and $+^{\mathcal{M}_{1}}$ are the interpretations of $\sim$ and + in $\mathcal{M}_{1}$. However, I will omit such superscripts if there is no risk of causing confusion. By the way, to show that $x+y=x \cup y$, first observe that $x \cup y$ can serve as the $z$ such that $\forall u(O(u, z) \leftrightarrow(O(u, x) \vee O(u, y)))$, but such a kind of $z$ must be unique in $\mathcal{M}_{1}$ since $\forall z(O(z, x) \rightarrow O(z, y)) \leftrightarrow P(x, y)$ is an easy theorem of $\mathbf{E M}=(\mathrm{P} 1)+(\mathrm{P} 2)+(\mathrm{P} 3)+(\mathrm{SSP})$ and $\mathcal{M}_{1}$ satisfies EM. The said easy theorem is very useful for proving the identity between two members in any structure which satisfies EM.

9. The so-called traditional way can be seen in [5], [11], and [8], as well as in many other prestigious textbooks for mathematical logic.

10. I am indebted to Haim Gaifman for inspiring me to use a "chain" to stand for a natural number. Actually I gave a sketch in my dissertation [17] several years ago of how to make use of "chains" to show that the theory of $\mathcal{M}_{1}$ is not axiomatizable. However, many things were not clear then, and the idea of the proof relies on the fact that there are "boundaries" in $\operatorname{Dom}\left(\mathcal{M}_{1}\right)$ and hence cannot apply to the case of $\mathcal{M}_{2}$.

11. This definition is attributed to Pratt-Hartmann ([10, p. 28]). He has also shown there that $x$ is self-connected in $\mathcal{M}_{2}$ if and only if $x$ is a connected open subset of $R^{2}$, that is, $x$ is not the union of two nonempty disjoint open subsets of $R^{2}$.

12. The following is a "canonical" sample of UFC in $\mathcal{M}_{2}$ : the union of the regular open disks which are defined by $(x-d)^{2}+(y-e)^{2}<1$, where $(d, e) \in\{(2 k, 4 m) \in \omega \times \omega$ : $m, k \in \omega$ and $k \leq m\}$.

13. The definitions of "essentially undecidable" and "hereditarily undecidable" are due to Tarski, Mostowski, and Robinson [13]. The definition of "strongly undecidable" is given by [5], which applies to logical theories, not to models. 


\section{References}

[1] Casati, R., and A. C. Varzi, Parts and Places: The Structures of Spatial Representation, MIT Press, Cambridge, Mass., 1999. 287, 288, 289, 304

[2] Ciraulo, F., M. E. Maietti, and P. Toto, "Constructive version of Boolean algebra," Logic Journal of the IGPL, vol. 21 (2012), pp. 44-62. MR 3020708. DOI 10.1093/jig$\mathrm{pal} / \mathrm{jzs} 021.303$

[3] Clarke, B. L., "A calculus of individuals based on "connection," Notre Dame Journal of Formal Logic, vol. 22 (1981), pp. 204-18. Zbl 0438.03032. MR 0614118. 290

[4] Düntsch, I., and E. Orłowska, "A proof system for contact relation algebras," Journal of Philosophical Logic, vol. 29 (2000), pp. 241-62. MR 1773955. DOI 10.1023/A:1004764610651. 304

[5] Enderton, H. B., A Mathematical Introduction to Logic, 2nd ed., Academic Press, Burlington, Mass., 2001. MR 1801397. 302, 305

[6] Leśniewski, S., "Foundations of the general theory of sets, I," (in Polish) in S. Leśniewski, Collected Works, Vol. 1, edited by S. J. Surma, J. T. Srzednicki, D. I. Barnett, and V. F. Rickey, Kluwer, Dordrecht, 1992. 288

[7] Libkin, L., Elements of Finite Model Theory, Springer, Berlin, 2004. Zbl 1060.03002. MR 2102513. DOI 10.1007/978-3-662-07003-1. 296

[8] Monk, J. D., Mathematical Logic, vol. 37 of Graduate Texts in Mathematics, Springer, New York, 1976. MR 0465767. 290, 305

[9] Munkres, J. R., Topology, 2nd ed., Prentice Hall, London, 2000. 305

[10] Pratt-Hartmann, I., "First-order mereotopology," pp. 13-97 in Handbook of Spatial Logics, edited by M. Aiello, I. Pratt-Hartmann, and J. van Benthem, Springer, Dordrecht, 2007. MR 2393886. DOI 10.1007/978-1-4020-5587-4_2. 287, 304, 305

[11] Shoenfield, J. R., Mathematical Logic, Addison-Wesley, London, 1967. MR 0225631. 295, 297, 302, 305

[12] Simons, P., Parts: A Study in Ontology, Clarendon Press, Oxford, 1987. 288

[13] Tarski, A., A. Mostowski, and R. M. Robinson, Undecidable Theories, North-Holland, Amsterdam, 1953. MR 0058532. 305

[14] Tsai, H., "Decidability of mereological theories," Logic and Logical Philosophy, vol. 18 (2009), pp. 45-63. Zbl 1186.03018. MR 2598306. DOI 10.12775/LLP.2009.004. 293

[15] Tsai, H., "A comprehensive picture of the decidability of mereological theories," Studia Logica, vol. 101 (2012), pp. 987-1012. DOI 10.1007/s11225-012-9405-z. 293

[16] Tsai, H., "Decidability of general extensional mereology," Studia Logica, vol. 101 (2013), pp. 619-36. DOI 10.1007/s11225-012-9400-4. 292, 293, 303

[17] Tsai, H., "The logic and metaphysics of part-whole relations," Ph.D. dissertation, Columbia University, New York, 2005. 305

[18] Whitehead, A. N., Process and Reality, MacMillan, New York, 1929. Zbl 55.0035.03. 304

\section{Acknowledgments}

This paper is a product of research projects kindly supported by National Science Council (Taiwan) grants NSC 99-2410-H-194-015-MY2 and NSC 101-2410-H-194-033MY3.

Department of Philosophy

National Chung-Cheng University

Chiayi 62102

Taiwan

pythc@ccu.edu.tw 